\title{
IL-7: AhR We Ready for a New Cytokine to Fight Colitis?
}

\author{
Atsuhito Nakao ${ }^{1,2}$
}

Published online: 22 May 2015

(c) Springer Science+Business Media New York 2015

The aryl hydrocarbon receptor (AhR) is a ligand-activated transcriptional factor ubiquitously expressed in vertebrate cells. AhR recognizes man-made synthetic compounds such as 2,3,7,8-tetrachlorodibenzo-p-dioxin (TCDD, dioxin) and naturally occurring compounds such as the tryptophan derivative indole-3-carbinol (I3C), which is present in cruciferous vegetables; AhR is thought to be involved in the metabolism or detoxification of these compounds [1]. Upon ligand binding, AhR translocates from the cytosol to the nucleus, dimerizes with the aryl hydrocarbon receptor nuclear translocator (AhRNT), and initiates the transcription of target genes with promoters containing the xenobiotic-responsive element (XRE) consensus sequence. The targets of this pathway include genes that encode xenobiotic-metabolizing enzymes, including cytochrome $\mathrm{P} 450$ family 1A1 (CYP1A1).

The AhR pathway not only functions in the metabolism of man-made and naturally occurring chemicals, but also regulates a variety of physiological processes [2]. In particular, this pathway acts as a crucial regulator of mucosal immunity in the gut [3]. Notably, there is accumulating evidence that activation of AhR is beneficial for colitis in mice and humans [4]. For instance, treatment with TCDD or AhR agonist 6-formylindolo(3, 2-b) carbazole (FICZ) protects mice against dextran sulfate sodium (DSS)-induced colitis $[5,6]$. In humans, intestinal $\mathrm{T}$ cells and

Atsuhito Nakao

anakao@yamanashi.ac.jp

1 Department of Immunology, Faculty of Medicine, University of Yamanashi, 1110 Shimokato, Chuo, Yamanashi 409-3898, Japan

2 Atopy Research Center, Juntendo University School of Medicine, Tokyo, Japan natural killer cells isolated from Crohn's disease patients express low levels of AhR [6]. Although the precise mechanisms remain to be determined, it is very likely that activation of the AhR in the gut ameliorates colitis by activating intestinal $\mathrm{ROR} \gamma \mathrm{t}^{+}$innate lymphoid cells (type 3 ILCs) to release the gut-protective cytokine IL-22 [7] or by augmenting the functions of intestinal intraepithelial lymphocytes (IELs) [8]. Both of these cell types are important for maintaining intestinal homeostasis.

In this issue of Digestive Diseases and Sciences, Ji et al. [9] have added a new and indispensable candidate, IL-7, to the list of factors involved in amelioration of colitis by the AhR pathway. Specifically, they reported that FICZ downregulates epithelial cell-derived IL-7 expression in mice with DSS-induced colitis, concomitant with amelioration of colitis, elevation of intestinal T cell receptor (TCR) $\gamma / \delta^{+}$IEL subpopulations, and reductions in intestinal-activated $\mathrm{CD} 4{ }^{+} \mathrm{CD}^{+}$IEL subpopulations. IL-7 plays an essential role in the development and homeostasis of T cells [10]. Recent studies also highlight the importance of IL-7 in intestinal homeostasis; specifically, IL-7 produced by intestinal epithelial cells is essential for the maintenance and functionality of colitogenic memory $\mathrm{T}$ cells and intestinal IELs [11, 12]. The identification of IL-7 as a novel target of the AhR pathway in the gut provides new insight into previously unknown aspects of the biology of the AhR pathway in the mucosal immune system. In light of these observations, it will be of interest to determine how environmental and dietary AhR ligands affect epithelial cell-derived IL-7 and influence the development and maintenance of mucosal $\mathrm{T}$ cells. In this context, future studies should attempt to reveal how FICZ or activation of the AhR pathway downregulates epithelial cell-derived IL-7 in the gut.

Direct activation of the AhR expressed in intestinal type 3 ILCs or IELs is important for amelioration of colitis [7, 
8]. Thus, the results of Ji et al. [9] raise the possibility that activation of the AhR pathway might alter intestinal type 3 ILCs or IELs functions indirectly, via modulation of epithelial cell-derived IL-7 expression, reducing intestinal inflammation. The findings of $\mathrm{Ji}$ et al. [9] suggest that modulation of the AhR pathway in intestinal epithelial cells, but not in intestinal type 3 ILCs and IELs, could become a primary target for amelioration of colitis via AhR activation (Fig. 1).

In addition to the specific questions mentioned above, it is important to determine to what extent changes in intestinal IEL phenotypes contribute to the amelioration of DSS-induced colitis. Furthermore, it remains uncertain how DSS-induced colitis upregulates IL-7 expression in intestinal epithelial cells. Moreover, it is not fully understood whether orally administered FICZ or other AhR ligands could recapitulate results similar to those of systemic administration of FICZ (i.e., downregulation of epithelial cell-derived IL-7). These issues should be also addressed in future studies.

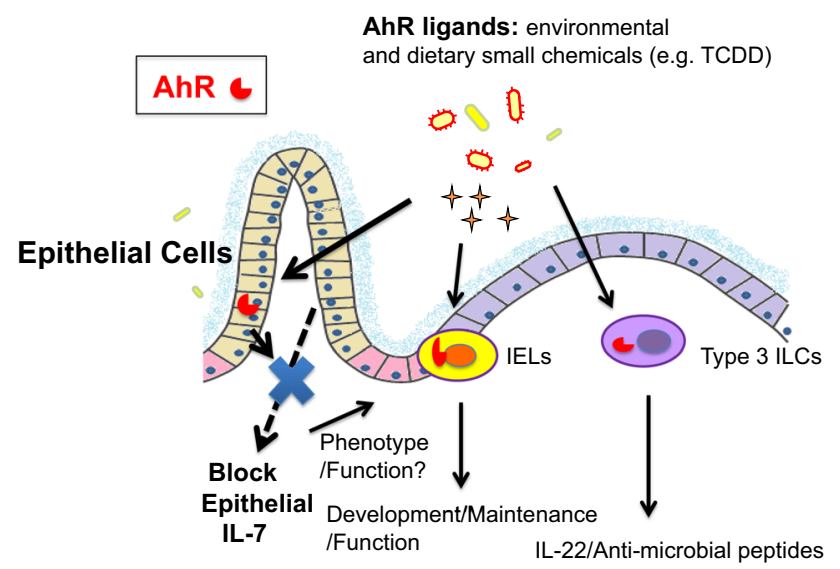

Suppression of colitis

Fig. 1 IL-7 may be a novel target for AhR-mediated suppression of colitis. Previous studies suggest that activation of AhR in the gut ameliorates colitis by stimulating intestinal $\mathrm{ROR} \gamma \mathrm{t}^{+}$innate lymphoid cells (type 3 ILCs) to release the gut-protective cytokine IL-22, or via the development (maintenance) and function of intestinal intraepithelial lymphocytes (IELs). Both of these cell types play important roles in maintaining intestinal homeostasis. Ji et al. [9] propose that epithelial cell-derived IL-7 is an important target for the AhRmediated suppression of colitis. Inhibition of IL-7 through AhR activation by AhR ligands such as FICZ could affect phenotypes and function of intestinal IELs, thereby suppressing experimental colitis in mice
The findings of Ji et al. [9] provide novel insight into the mechanism by which activation of the AhR pathway ameliorates intestinal inflammation; IL-7 blockade may mediate the beneficial effects of AhR pathway activation on colitis. Likewise, this study suggests that the AhR pathway may be more influential in the homeostatic regulation of mucosal immune system, in particular $\mathrm{T}$ cell (and possibly also B cell) development and function in the intestine, than previously thought. Studies aimed at elucidating the link between the AhR pathway and mucosal immune system will eventually inform the development of new methods to prevent and treat inflammatory bowel diseases using AhR ligands and agonists.

\section{References}

1. Hankinson O. The aryl hydrocarbon receptor complex. Annu Rev Pharmacol Toxicol. 1995;35:307-340.

2. Barouki R, Coumoul X, Fernandez-Salguero PM. The aryl hydrocarbon receptor, more than a xenobiotic-interacting protein. FEBS Lett. 2007;581:3608-3615.

3. Esser C, Rannug A, Stockinger B. The aryl hydrocarbon receptor in immunity. Trends Immunol. 2009;30:447-454.

4. Monteleone I, Macdonald TT, Pallone F, Monteleone G. The aryl hydrocarbon receptor in inflammatory bowel disease: linking the environment to disease pathogenesis. Curr Opin Gastroenterol. 2012;28:310-313.

5. Takamura T, Harama D, Matsuoka S, et al. Activation of the aryl hydrocarbon receptor pathway may ameliorate dextran sodium sulfate-induced colitis in mice. Immunol Cell Biol. 2010;88:685-689.

6. Monteleone I, Rizzo A, Sarra M, et al. Aryl hydrocarbon receptor-induced signals up-regulate IL-22 production and inhibit inflammation in the gastrointestinal tract. Gastroenterology. 2011;141:237-248.

7. Qiu J, Heller JJ, Guo X, et al. The aryl hydrocarbon receptor regulates gut immunity through modulation of innate lymphoid cells. Immunity. 2012;36:92-104.

8. Li Y, Innocentin S, Withers DR, et al. Exogenous stimuli maintain intraepithelial lymphocytes via aryl hydrocarbon receptor activation. Cell. 2011;147:629-640.

9. Ji T, Xu C, Sun L, et al. Aryl hydrocarbon receptor activation down-regulates IL-7 and reduces inflammation in a mouse model of DSS-induced colitis. Dig Dis Sci. (Epub ahead of print). doi:10.1007/s10620-015-3632-x.

10. Boyman O, Krieg C, Homann D, Sprent J. Homeostatic maintenance of T cells and natural killer cells. Cell Mol Life Sci. 2012;69:1597-1608.

11. Kanai T, Nemoto Y, Kamada N, et al. Homeostatic (IL-7) and effector (IL-17) cytokines as distinct but complementary target for an optimal therapeutic strategy in inflammatory bowel disease. Curr Opin Gastroenterol. 2009;25:306-313.

12. Yang H, Madison B, Gumucio DL, Teitelbaum DH. Specific overexpression of IL-7 in the intestinal mucosa: the role in intestinal intraepithelial lymphocyte development. Am J Physiol Gastrointest Liver Physiol. 2008;294:G1421-G1430. 